\title{
figh-Gain, Very Low Side-Lobe Antenna With Capability for Beam Slewing ${ }^{1,2}$
}

\author{
Alvin C. Wilson
}

\author{
(October 8, 1959; revised March 21, 1960)
}

\begin{abstract}
A corner-reflector antenna having reflecting surfaces ten wavelengths wide and two wavelengths long was constructed, adjusted, and tested. The driven element was a collinear array of ten half-wave dipoles. Dolph-Chebyshev current distribution designed for side-lobe suppressions to -45 decibels was computed. The currents in the dipoles were adjusted as nearly as possible to this distribution. The phase of the dipole currents was graded so as to slew the main beam $10^{\circ}$ off the forward direction. The radiation patterns were measured and found to be quite close to the computed.

Check of pattern stability with time and with changes in temperature and weather conditions showed it to be quite stable. Measurements of radiation pattern at frequencies departing from design frequency showed the operating bandwidth (determined by the preservation of the pattern) to be adequate for the applications likely to be considered for such antennas.

The half-power beam widths of the main lobe were $9.8^{\circ}$ in the $E$-plane and $32^{\circ}$ in the $H$-plane. On the basis of the measured beamwidths, the gain was calculated to be approximately 21.2 decibels relative to an isotropic radiator. The gain was experimentally measured to be 21.2 decibels.
\end{abstract}

\section{Introduction}

Operation of VHF ionospheric scatter communiation services at frequencies below the $F 2$ MUF equires, especially for high speed binary data ransmission, special means to avoid long range nterference, including self-interference from backcatter $[1] .^{3}$ The use of narrow beam antennas lesigned to suppress the radiation outside the main obe by at least $40 \mathrm{db}$ has been indicated as one neasure for reducing such interference. In special iting situations, severe requirements occasionally rise for suppression of radiation outside the main lobe is a means of avoiding interference to neighboring adio services. Under some circumstances, there vould be an advantage in having an antenna in which the main beam could be slewed to either side or taking advantage of meteor reflections.

The performance of corner-reflector antennas recame of interest to the National Bureau of Standrds because they appeared to be well suited to ommunication via ionospheric scatter. Previous neasurements have shown that this antenna can be esigned to have a high gain with very low secondary obe-levels $[2,3]$. A collinear array of dipoles may se used in a corner-reflector antenna to obtain ligher gain and more desirable directive characterstics than are obtainable by the use of a single lipole in a corner reflector.

A method of determining the optimum current distrisution in a collinear array of isotropic radiators has seen described by C. L. Dolph [4]. The resultant

1 Contribution from Central Radio Propagation Laboratory, National Bureau f Standards, Boulder, Colo.

2 The work reported herein was carried out on behalf of the U.S. Air Force nder support extended by U.S. Air Force Ground Electronics Engineering and nstallation Agency, Rome, N.Y.

3 Figures in brackets indicate the literature references at the end of this paper. current distribution across the array is based on the properties of Chebyshev polynomials. When a Dolph-Chebyshev current distribution is used, the main beamwidth will be as narrow as possible for a predetermined side-lobe level; or, if the main beamwidth is specified, the side-lobe level will be a minimum.

When a collinear array of dipoles is used in a corner reflector, the resultant pattern is more directive than the array pattern for the collinear dipoles alone, and further reduction in levels of side lobes is achieved.

Computations were made to obtain the DolphChebyshev current distributions to limit the side lobes $40 \mathrm{db}$ below the main beam level and the resultant half-power beamwidth for collinear arrays of $6,8,10,12,14$, and 16 half-wave dipoles spaced 0.5 and 0.8 wavelengths apart. Table 1 shows the relative current distribution and resultant main beam half-power beamwidth.

TABLE 1. Computed dipole current distribution and resultant half-power beamwidth of main lobe for Dolph-Chebyshev current distribution to limit all side lobes 40 db below main beam level

\begin{tabular}{|c|c|c|c|c|c|c|c|c|c|c|}
\hline \multirow{3}{*}{$\begin{array}{l}\text { Number } \\
\text { of } \\
\text { dipoles } \\
\text { in } \\
\text { collinear } \\
\text { array }\end{array}$} & \multicolumn{2}{|c|}{$\begin{array}{l}\text { Half-power beam- } \\
\text { width in degrees }\end{array}$} & \multirow{2}{*}{\multicolumn{8}{|c|}{$\begin{array}{l}\text { Current distribution, decibels of attenuation } \\
\text { relative to center elements } \\
\text { Dipole right and left from array center }\end{array}$}} \\
\hline & \multirow{2}{*}{$\begin{array}{c}0.5 \lambda \\
\text { spacing } \\
\text { between } \\
\text { dipole } \\
\text { centers }\end{array}$} & \multirow{2}{*}{$\begin{array}{c}0.8 \lambda \\
\text { spacing } \\
\text { between } \\
\text { dipole } \\
\text { centers }\end{array}$} & & & & & & & & \\
\hline & & & 1 & 2 & 3 & 4 & 5 & 6 & 7 & 8 \\
\hline 6 & 23.6 & 15.0 & 0 & 4. 2 & 14.0 & & & & & \\
\hline 8 & 18.0 & 11. 4 & 0 & 2.4 & 7. 6 & 16.7 & & & & \\
\hline 10 & 14.5 & 9.2 & 0 & 1.5 & 4. 7 & 10.0 & 18. 1 & & & \\
\hline 12 & 12.1 & 7. 6 & 0 & 1.05 & 3.2 & 6.7 & 11.8 & 18.6 & & \\
\hline 14 & 10.3 & 6.4 & 0 & 0.76 & 2.3 & 4.8 & 8. 3 & 13.1 & 18.9 & \\
\hline 16 & 9.0 & 5.6 & 0 & .58 & 1.8 & 3.6 & 6.2 & 9.6 & 14.2 & 18. 9 \\
\hline
\end{tabular}


In order to slew the main beam of a collinear array away from the forward direction, a change in the phase of current in the dipoles may be calculated for the desired amount of slewing.

The phase advance $\Phi_{p}$ of the current in a dipole $p$ required to produce a slew angle $\beta_{0}$ off the broadside direction is given in degrees by

$$
\Phi_{p}=-360 \frac{d p}{\lambda} \sin \beta_{0}
$$

where $d p$ is the distance of the dipole from the center of the array, taken as zero reference for phase. The negative sign signifies that the phase is retarded when the slew angle is in the direction of positive $p$.

Computations were made to obtain the radiation patterns for a collinear array of 16 half-wavelength dipoles, spaced a half-wavelength apart with the main beam slewed $0^{\circ}, 10^{\circ}$, and $20^{\circ}$ off from the forward direction. It was noted that, even with the beam slewed as much as $20^{\circ}$, the side lobes were still nearly $40 \mathrm{db}$ down from the main beam level. Figure 1 presents these computed patterns.

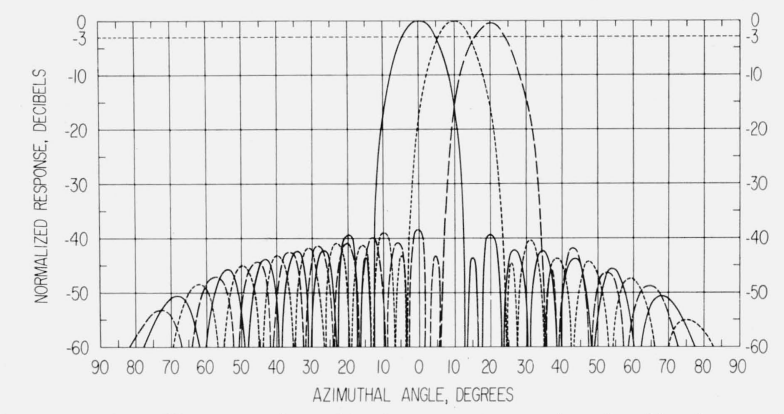

Figure 1. Computed radiation patterns in the E-plane of a collinear array of 16 half-wavelength dipoles spaced halfwavelength apart with a Dolph-Chebyshev current distribution computed to limit the side lobes below $40 \mathrm{db}$.

- Maximized in forward direction.
$\ldots \ldots \ldots$ Beam maximum $10^{\circ}$ off forward direction.

The radiation patterns for a collinear array of 10 half-wavelength dipoles spaced $0.8 \lambda$ apart in a corner reflector was computed. The aperture angle of the corner reflector was assumed to be $90^{\circ}$ for purposes of computation, and the dipole position, distance to apex, 0.3 wavelength. Using a dipole current distribution to limit the side lobes $40 \mathrm{db}$ below the main beam level, a main beamwidth at the halfpower points of $9.2^{\circ}$ was obtained. When the beam of this corner-reflector antenna is slewed by grading the phase of the dipole currents, the side lobes will not all be $40 \mathrm{db}$ below the main beam level. With the beam slewed $10^{\circ}$, a high side lobe occurs at an azimuth of $68^{\circ}$ in the direction opposite from slew; its level is $37 \mathrm{db}$ below the main beam level. With the beam slewed $20^{\circ}$, the corresponding side lobe occurs at $56^{\circ}$ with the response $17 \mathrm{db}$ down from the main beam level. The high side lobes are actually secondary main lobes due to the dipole spacing being greater than a half-wavelength. Figure 2 shows the computed patterns for $0^{\circ}, 10^{\circ}$, and $20^{\circ}$ slew.

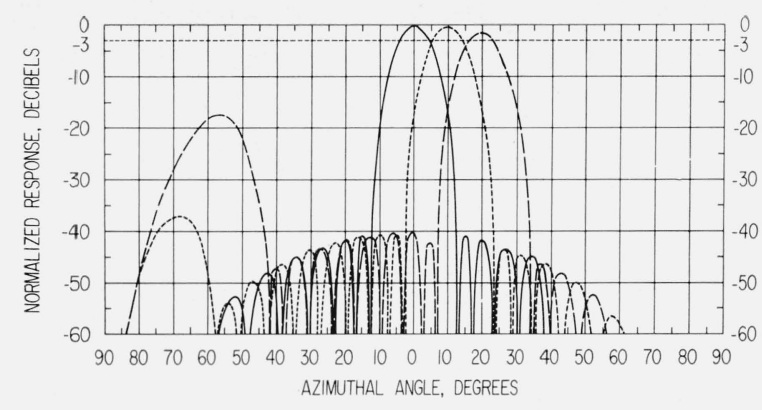

FIGURE 2. Computed radiation patterns of a $90^{\circ}$ cornerreflector antenna using a collinear array of 10 half-wavelength dipoles as the driven element.

Dipoles spaced $0.8 \lambda$ apart and $0.3 \lambda$ from the apex. The dipcle currents follow Dolph-Chebyshev distribution computed to limit the side lobes below $40 \mathrm{db}$. Maximized in forward direction.
$-\ldots \ldots$ Beam maximum $10^{\circ}$ off forward direction.

A corner-reflector type of antenna with a DolphChebyshev dipole current distribution was constructed, adjusted, and tested by the Boulder Laboratories of the National Bureau of Standards. The adjustments and tests were performed in two phases. The work performed in phase I consisted of the adjustments and measurements necessary to obtain side-lobe suppressions below $-40 \mathrm{db}$. The work performed in phase II consisted of the adjustments and measurements necessary to slew the main beam $10^{\circ}$ in addition to side-lobe suppressions below $-40 \mathrm{db}$. Gain measurements were made for the antenna in both test phases.

The terminology employed here conforms to com mon usage. The width of the reflecting surfaces, $W$, is the dimension parallel to the apex line. The length, $L$, is measured along the direction normal to the apex line. The aperture angle, $\theta$, is that formed by the two reflecting surfaces. The dipole position, $S$, is the distance of the driven element from the apex. The dipole spacing is measured between the dipole centers.

\section{Experimental Procedure}

On the basis of gains and radiation patterns obtained with corner-reflector structures havino various combinations of lengths and widths of reflecting surfaces $[2,3]$ and on the basis of the computed radiation patterns for collinear arrays, a corner-reflector structure with reflecting surfaces 2 wavelengths long and 10 wavelengths wide and an aperture angle of $72^{\circ}$ was constructed, adjusted and tested with a Dolph-Chebysher dipole curren distribution to limit the side-lobe level.

The driven element was a collinear array of 10 half-wave dipoles spaced 0.8 wavelength center-to center from each other and 0.45 wavelength from the apex of the corner-reflector structure.

The currents in the dipoles were initially adjustea to conform to the values determined for a Dolph Chebyshev current distribution to limit the side lobes to $-40 \mathrm{db}$. It was found, however, that smal departures in current values, unavoidable in experi- 
probably a function of the corner-reflector structure. No further attempt was made to reduce these back-radiation lobes since they were already below the secondary lobe level at $110^{\circ}$. Except for a small side lobe at the base of the main lobe, all lobes in the vicinity of the main beam are below $-40 \mathrm{db}$ and even approach $-45 \mathrm{db}$.

Measurements were carried out to determine the degree of cross polarization. Figure 5 presents these results. The curve shown as the solid line is a normal $E$-plane radiation pattern. The curve shown

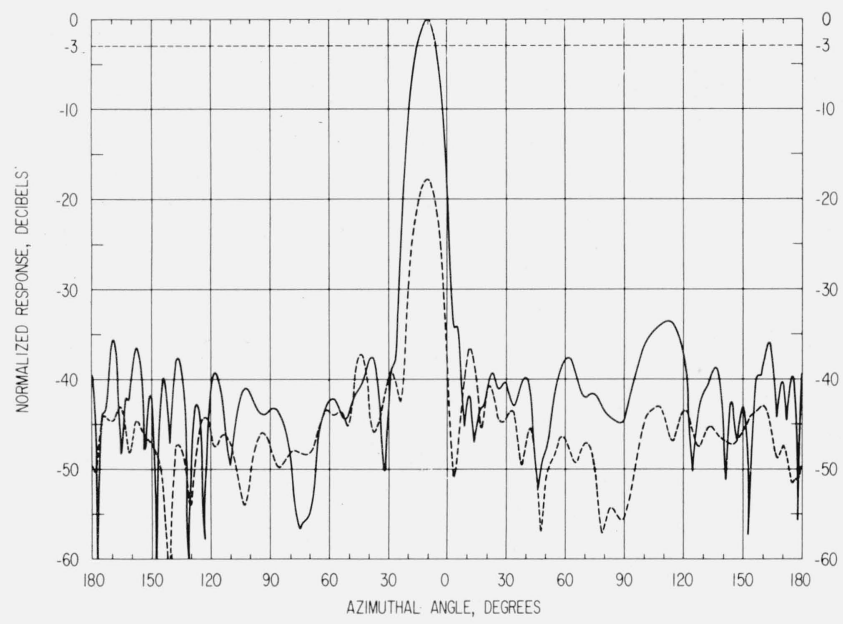

FiguRe 5. Radiation pattern in the E-plane and the cross polarization pattern in the same plane with the main beam slewed $10^{\circ}$ off the forward direction.

Normal E-plane pattern.

$\ldots \ldots$. Cross polarization pattern.

as the dashed line shows the cross polarization in the same plane. The main lobe of the cross polarization pattern is seen to be approximately $18 \mathrm{db}$ below that of the normal pattern.

Measurements were made of the frequency sensitivity of the radiation pattern by taking patterns at $0.2-\mathrm{Mc} / \mathrm{s}$ intervals above and below the design frequency of $400 \mathrm{Mc} / \mathrm{s}$. Figure 6 presents these patterns. As the frequency deviation became greater, the level of the side lobes near the base of the main beam increased. There was no measurable change in the back radiation or in the degree of beam slew.

Of considerable importance is the problem of electrical stability of the system with time. While the experimental measurements described were not designed with stability as the prime objective, measurements have been made on the antenna with adjustments left unchanged with time. Figure 7 shows radiation patterns recorded on three different days with adjustments not disturbed. The slight day-today changes appeared to be caused by weather conditions and temperature differences.

On the basis of the half-power beamwidths obtained for the $H$-plane from the radiation patterns of phase I and the $E$-plane from radiation patterns of phase II, the gain of the antenna over an isotropic radiator may be computed from the following [6]:

$$
\begin{aligned}
& \text { Gain }(\mathrm{db})=10 \log _{10} \frac{41,253}{W_{E} W_{H}} \\
& \text { gain }(\mathrm{db})=10 \log _{10} \frac{41,253}{9.8 \times 32}
\end{aligned}
$$$$
\text { gain }(\mathrm{db})=21.2 \text { 。 }
$$

This method of estimating the gain is most accurate when the main beam is narrow and the side lobes are minimum as is the case for this antenna. The gain was experimentally measured relative to a half-wave dipole. These measurements indicate a gain of $21.2 \mathrm{db}$ over an isotropic radiator. This gair measurement was an average of three experimental measurements with maximum deviation $\pm 0.5 \mathrm{db}$.

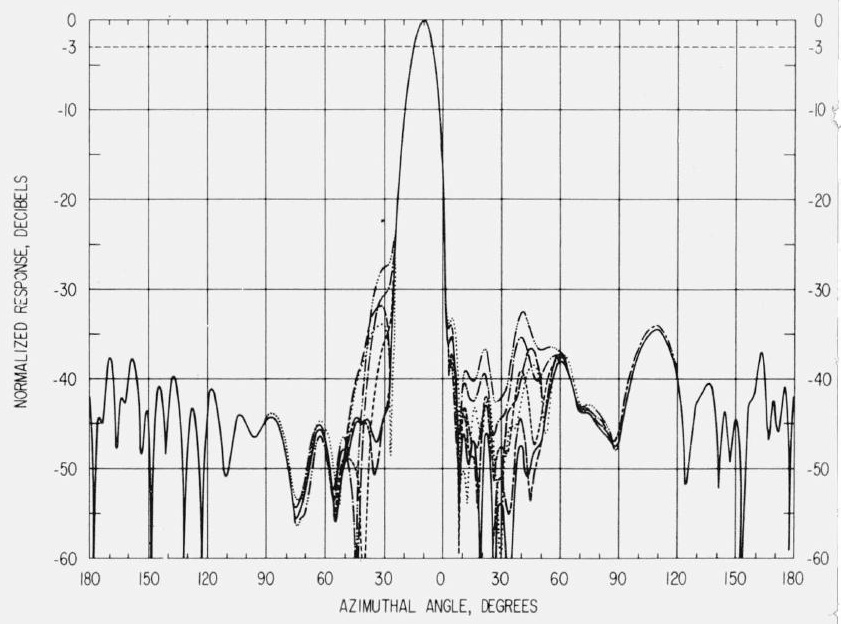

FIGURE 6. Effect of frequency changes on the radiation pattern.
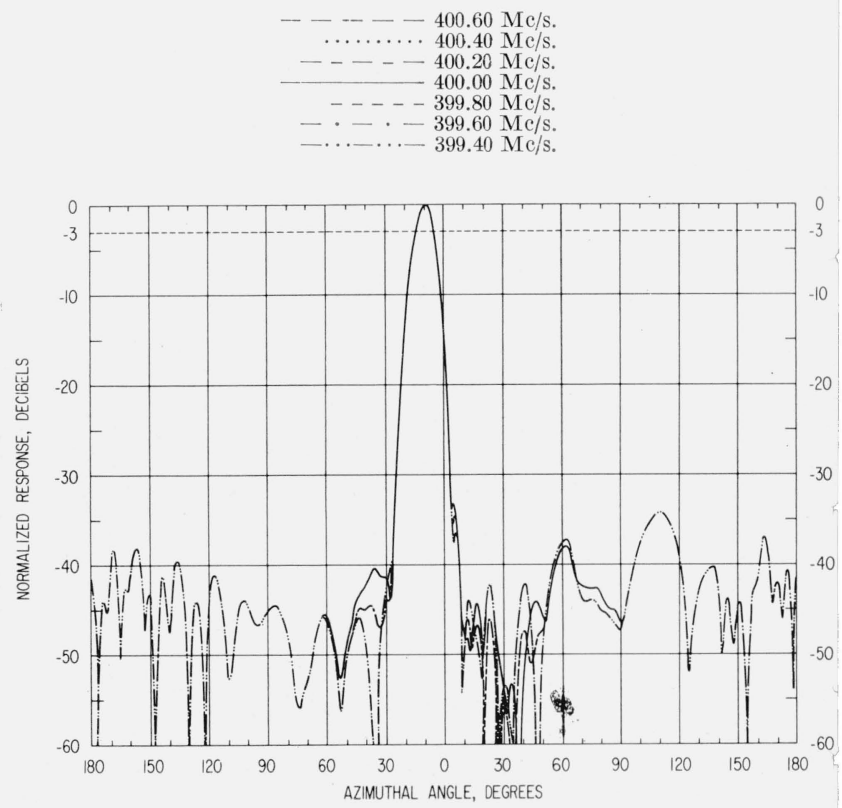

Figure 7. Stability of radiation pattern with time.

Radiation pattern taken December 16, 1958. _......... Radiation pattern taken December 18, 1958. 


\section{Conclusions}

The experimental work described shows that it is possible to approach in practice the results obtained theoretically for side-lobe suppression and beam slewing by using an array of collinear dipoles in a cornerreflector antenna. Compensation can be made for deviations of actual dipole currents from the theoretical currents by using a current distribution for $-45 \mathrm{db}$ to obtain in practice -40 - $\mathrm{db}$ side-lobe suppression.

The corner-reflector antenna using a collinear array of dipoles as the driven element has been found to be a suitable antenna type for applications requiring a high gain, low secondary radiation levels, and a capability for beam slewing.

Beam slewing of more than $10^{\circ}$ is practical, depending upon the tolerance for a secondary lobe at a large angle from the main lobe. To reduce this difficulty, the dipole spacing may be reduced. At $0.5 \lambda$ dipole spacing, the secondary lobe is effectively eliminated, but mutual coupling between dipoles increases the problems of adjustment of current distribution and phase.

Full-scale operational antennas of this type are practical to construct and maintain. The desired degrees of beam slew can be obtained by the use of properly adjusted plug-in current distribution networks. For purposes of initial adjustment and subsequent monitoring, a current sampling loop should be built into each dipole.

The measurements were carried out with the assistance of W. L. Martin.

\section{References}

[1] R. C. Kirby, 1958 critique of VHF ionospheric scatter communication, Symp. Record, IRE-GW U Natl. Symp. on Extended Range and Space Commun. (1958).

[2] H. V. Cottony and A. C. Wilson, Gains of finite-size corner-reflector antennas, IRE Trans. on Antennas and Propagation AP-6, 336 (1958)

[3] A. C. Wilson and H. V. Cottony, Radiation patterns of finite-size corner-reflector antennas, IRE Trans. on Antennas and Propagation AP-8, 144 (1960).

[4] C. L. Dolph, A current distribution for broadside arrays which optimize the relationship between beam width and side-lobe level, Proc. IRE 34, 335 (1946).

[5] H. V. Cottony, Techniques for accurate measurement of antenna gain, NBS Circ. 598 (1958).

(Paper 64D5-93) 